\title{
Chiral anomaly and strange-nonstrange mixing
}

\author{
Francesco Giacosa ${ }^{1,2, \star}$ \\ ${ }^{1}$ Institute of Physics, Jan Kochanowski University, ul. Swietokrzyska 15, Kielce, Poland \\ ${ }^{2}$ Institute for Theoretical Physics, J.W. Goethe University, Max-von-Laue-Str. 1, Frankfurt am Main, Germany
}

\begin{abstract}
As a first step, a simple and pedagogical recall of the $\eta-\eta^{\prime}$ system is presented, in which the role of the axial anomaly, related to the heterochiral nature of the multiplet of (pseudo)scalar states, is underlined. As a consequence, $\eta$ is close to the octet and $\eta^{\prime}$ to the singlet configuration. On the contrary, for vector and tensor states, which belong to homochiral multiplets, no anomalous contribution to masses and mixing is present. Then, the isoscalar physical states are to a very good approximation nonstrange and strange, respectively. Finally, for pseudotensor states, which are part of an heterochiral multiplet (just as pseudoscalar ones), a sizable anomalous term is expected: $\eta_{2}(1645)$ roughly corresponds to the octet and $\eta_{2}(1870)$ to the singlet.
\end{abstract}

\section{Introduction}

The meson $\eta^{\prime} \equiv \eta^{\prime}(958)$ is special: its large mass and its flavor content are strongly influenced by the so-called axial anomaly [1-3] (the classical $U(1)_{A}$ symmetry of QCD is broken by quantum fluctuations). Roughly speaking, $\eta^{\prime}$ corresponds to a flavor singlet, while $\eta \equiv \eta(547)$ to the octet. In Sec. 1, we recall some basic features of the $\eta-\eta^{\prime}$ and we connect them to the heterochirality $[4,5]$ of pseudoscalar states and their chiral partners, the scalar states.

A natural question is if the axial anomaly affects other mesons. Interestingly, it turns out that the axial anomaly does not affect the vector states $\omega(782)$ and $\phi(1020)$ and the tensor states $f_{2}(1270)$ and $f_{2}^{\prime}(1525)$ (see Sec. 3): $\omega(782)$ and $f_{2}(1270)$ are (almost purely) nonstrange and $\phi(1020)$ and $f_{2}^{\prime}(1525)$ strange. This fact can be nicely understood by the homochirality of the corresponding chiral multiplets, which involve left- and right-handed currents. For homochiral multiplets, no anomalous mixing is realized [4].

Are there other mesons for which the anomaly plays a role? This seems to be the case of pseudotensor mesons (Sec. 4). As shown in the phenomenological study of Ref. [6], the mesons $\eta_{2}(1645)$ and $\eta_{2}(1870)$ roughly correspond to octet and singlet states (the mixing angle is similar to the one of $\eta$ and $\eta^{\prime}$ ). The pseudotensor mesons belong to a heterochiral multiplet (just as pseudoscalar states), hence one can understand why the axial anomaly is relevant.

\section{Pseudoscalar sector}

First, we review some features of the pseudoscalar sector. We consider the strange-nonstrange basis $\eta_{N}=\sqrt{1 / 2}(\bar{u} u+\bar{d} d), \eta_{S}=\bar{s} s$ and the octet-singlet basis $\eta_{8}=\sqrt{1 / 6}(\bar{u} u+\bar{d} d-2 \bar{s} s), \eta_{0}=\sqrt{1 / 3}(\bar{u} u+$

^fgiacosa@ujk.edu.pl 
$\bar{d} d+\bar{s} s$ ). The physical fields $\eta \equiv \eta(547)$ and $\eta^{\prime} \equiv \eta^{\prime}(958)$ are a mixture of $\eta_{N}$ and $\eta_{S}$ (and, similarly, of $\eta_{0}$ and $\eta_{8}$ ), according to:

$$
\left(\begin{array}{c}
\eta \\
\eta^{\prime}
\end{array}\right)=\left(\begin{array}{cc}
\cos \theta_{P} & \sin \theta_{P} \\
-\sin \theta_{P} & \cos \theta_{P}
\end{array}\right)\left(\begin{array}{l}
\eta_{N} \\
\eta_{S}
\end{array}\right),\left(\begin{array}{l}
\eta_{0} \\
\eta_{8}
\end{array}\right)=\left(\begin{array}{cc}
\sqrt{2 / 3} & \sqrt{1 / 3} \\
\sqrt{1 / 3} & -\sqrt{2 / 3}
\end{array}\right)\left(\begin{array}{l}
\eta_{N} \\
\eta_{S}
\end{array}\right) .
$$

The determination of $\theta_{P}$ is one important aspect of the problem.

We now introduce the Lagrangian terms for masses and mixing. The flavour invariant term is simply given by:

$$
\mathcal{L}_{P, U(3)}=-\frac{m_{P}^{2}}{2}\left(\eta_{N}^{2}+\eta_{S}^{2}\right)=-\frac{m_{P}^{2}}{2}\left(\eta_{0}^{2}+\eta_{8}^{2}\right)
$$

After spontaneous symmetry breaking $m_{P}^{2} \propto\left(m_{u}+m_{d}\right) / 2$, see e.g. Ref. [7]. If only $\mathcal{L}_{P, U(3)}$ is taken into account, one could use $\eta_{0}-\eta_{8}$ or $\eta_{N}-\eta_{S}$ (the octet-singlet choice is mathematically preferable). Next, the fact that the $s$-quark is more massive than the quarks $u$ and $d$ is taken into account by the Lagrangian

$$
\mathcal{L}_{P, S}=-\frac{\delta_{P, S}}{2} \eta_{S}^{2}
$$

with $\delta_{P, S}=2\left(m_{K}^{2}-m_{\pi}^{2}\right)\left(m_{K}\right.$ and $m_{\pi}$ are the kaon and pion masses). If $\mathcal{L}_{U(3)}+\mathcal{L}_{S}$ is considered, the physical states are $\eta_{N}$ (with squared mass $m_{P}^{2}$ ) and $\eta_{S}$ (with squared mass $m_{P}^{2}+\delta_{S}$ ). Last, the octet-singlet splitting is parametrized by

$$
\mathcal{L}_{P, 0}=-\alpha_{P} \eta_{0}^{2}=-\alpha_{P}\left(\sqrt{2} \eta_{N}+\eta_{S}\right)^{2},
$$

where $\alpha_{P}=\alpha_{P, g g}+\alpha_{P, A}$. Here, $\alpha_{P, g g}$ describes processes with two intermediate transverse gluons $\left(\bar{n} n \rightarrow \bar{n} n, \bar{n} n \rightarrow \bar{s} s\right.$, etc. ). This is a small perturbation. The parameter $\alpha_{P, A}$ represents an effective contribution of the axial anomaly; Eq. (4) with $\alpha_{P} \simeq \alpha_{P, A}$ was also obtained in e.g. Refs. [8, 9]. If $\mathcal{L}_{P, U(3)}+\mathcal{L}_{P, 0}$ is considered, the physical states are $\eta_{8}$ (with squared mass $m_{P}^{2}$ ) and $\eta_{0}$ (with squared mass $m_{P}^{2}+2 \alpha_{P}$ ). Thus, $\mathcal{L}_{P, S}$ and $\mathcal{L}_{P, 0}$ lead to different basis, and the question is which one is dominant.

In the full case, one considers $\mathcal{L}_{U(3)}+\mathcal{L}_{S}+\mathcal{L}_{P, 0}$. The pseudoscalar mixing angle $\theta_{P}$ can be calculated by the previous expressions: $\theta_{P}=-\frac{1}{2} \arctan \left[\frac{4 \sqrt{2} \alpha_{P}}{2\left(m_{K}^{2}-m_{\pi}^{2}-\alpha_{P}\right)}\right]$. Numerically, $\theta_{P}$ varies between $-40^{\circ}$ and $-45^{\circ}[2,3,7,10]$. The mixing is rather large and the states are closer to octet and singlet ones, but the effect of the $s$-quark is also important. Note, in the limit $\alpha_{P}=0$ one gets $\theta_{P}=0$ (purely strange and nonstrange states). On the contrary, in the limit $m_{K}^{2}-m_{\pi}^{2}=0\left(\delta_{P, S}=0\right)$ one has $\theta_{P}=\frac{1}{2} \arctan [2 \sqrt{2}]=35.3^{\circ}$, i.e. octet and singlet states, see Eq. (1).

In the recent work of Ref. [4], it was shown that the (pseudo)scalar multiplet is heterochiral. Namely, it is described by a matrix $\Phi$ (see [7]) which under chiral transformation changes as $\Phi \rightarrow e^{-i \alpha} U_{L} \Phi U_{R}^{\dagger}$ (the parameter $\alpha$ refers to $\left.U(1)_{A}\right)$. The Lagrangian $\mathcal{L}_{\Phi}^{\text {anomaly }}=-a_{\mathrm{A}}^{(3)}[\operatorname{det}(\Phi)-$ $\left.\operatorname{det}\left(\Phi^{\dagger}\right)\right]^{2}$ preserves chiral symmetry but breaks $U(1)_{A}$ (this is a consequence of the determinant, see also Ref. [5]). This Lagrangian term reduces to Eq. (4) when condensation is considered and quadratic mass terms are isolated. In conclusion, the heterochiral (pseudo)scalar nonet can easily explain the emergence of an anomalous term affecting $\eta$ and $\eta^{\prime}$.

\section{Vector (and tensor) mesons}

Next, we consider the isoscalar vector states $\omega(782)$ and $\phi(1020)$. Just as before, one introduces the nonstrange-strange basis $\omega_{N}=\sqrt{1 / 2}(\bar{u} u+\bar{d} d), \omega_{S}=\bar{s} s$ and the octet-singlet basis $\omega_{8}=\sqrt{1 / 6}(\bar{u} u+$ 
$\bar{d} d-2 \bar{s} s), \omega_{0}=\sqrt{1 / 3}(\bar{u} u+\bar{d} d+\bar{s} s)$, for which Eq. (1) holds (upon, of course, renaming the fields). Also here, we consider three Lagrangians:

$$
\mathcal{L}_{V, U(3)}=-\frac{m_{V}^{2}}{2}\left(\omega_{N}^{\mu 2}+\omega_{S}^{\mu 2}\right), \mathcal{L}_{V, S}=-\frac{\delta_{V, S}}{2} \omega_{S}^{\mu 2}, \mathcal{L}_{V, 0}=-\alpha_{V} \omega_{0}^{2}
$$

There is an important difference in the last term. For vector states, the constant $\alpha_{V}=\alpha_{V, g g g}$ (threegluon mixing processes, typically small): there is no contribution from the axial anomaly, $\alpha_{V, A}=0$. As a consequence, $\omega(782)$ is basically nonstrange and $\phi(1020)$ strange (mixing angle $\theta_{V}=-3^{\circ}$, equation analogous to Eq. (1) [11]). Similarly, for their axial-vector chiral partners it holds that: $f_{1}(1285)$ is almost purely nonstrange and $f_{1}(1420)$ purely strange [12].

In Ref. [4] it was discussed why $\alpha_{V, A}=0$. This is due to the fact that the corresponding chiral multiplets of vector $\left(V_{\mu}\right)$ and axial-vector $\left(A_{\mu}\right)$ states are homochiral. Namely, they enter into the $\operatorname{right}\left(\right.$ left)-handed $R_{\mu}=V_{\mu}-A_{\mu}$ and $L_{\mu}=V_{\mu}+A_{\mu}$, which under chiral symmetry transforms as $L_{\mu} \longrightarrow U_{\mathrm{L}} R_{\mu} U_{\mathrm{L}}^{\dagger}, R_{\mu} \longrightarrow U_{\mathrm{R}} R_{\mu} U_{\mathrm{R}}^{\dagger}$ (in both cases, either only $U_{\mathrm{L}}$ or $U_{\mathrm{R}}$ appears, but no mixed terms). There is no term involving the determinant.

A similar analysis applies to the ground-state tensor mesons, which are also part of an heterochiral multiplet: $f_{2}(1270)$ is almost purely nonstrange and $f_{2}^{\prime}(1525)$ strange, in agreement with the phenomenology [13].

\section{Pseudotensor mesons}

In the end, we consider the pseudotensor sector. We start from $\eta_{2, N}=\sqrt{1 / 2}(\bar{u} u+\bar{d} d), \eta_{S}=\bar{s} s$ and $\eta_{2,8}=\sqrt{1 / 6}(\bar{u} u+\bar{d} d-2 \bar{s} s), \eta_{2,0}=\sqrt{1 / 3}(\bar{u} u+\bar{d} d+\bar{s} s)$. The Lagrangian terms read

$$
\mathcal{L}_{P T, U(3)}=-\frac{m_{P T}^{2}}{2}\left(\eta_{2, N}^{\mu \nu, 2}+\eta_{2, S}^{\mu \nu, 2}\right), \mathcal{L}_{P T, S}=-\frac{\delta_{S}}{2} \eta_{2, S}^{\mu \nu 2}, \mathcal{L}_{P T, 0}=-\alpha_{P T} \eta_{2,0}^{\mu \nu, 2}
$$

Here, $\alpha_{P T}=\alpha_{P T, g g}+\alpha_{P T, A}$, and the latter quantity is expected to be sizable, hence the anomaly is potentially large. This is due to the fact that the corresponding chiral multiplet $\Phi_{\mu \nu}$ is heterochiral, just as for pseudoscalar mesons. In fact, under chiral transformations it transforms as $\Phi_{\mu \nu} \rightarrow \mathrm{e}^{-\mathrm{i} \alpha} U_{\mathrm{L}} \Phi_{\mu \nu} U_{\mathrm{R}}^{\dagger}$ [4]. The corresponding Lagrangian term $\mathcal{L}_{\Phi_{\mu v}}^{\text {anomaly }} \propto\left(\varepsilon^{i j k} \varepsilon^{i^{\prime} j^{\prime} k^{\prime}} \Phi^{i i^{\prime}} \Phi^{j j^{\prime}} \Phi_{\mu \nu}^{k k^{\prime}}-\text { h.c. }\right)^{2}$ is chirally symmetric but breaks $U(1)_{A}$ (it is an extension of the determinant) and reduces to $\mathcal{L}_{P T, 0}$ when the condensation of $\Phi$ is considered. The physical fields $\eta_{2}(1645)$ octet, $\eta_{2}(1870)$ are:

$$
\left(\begin{array}{l}
\eta_{2}(1645) \\
\eta_{2}(1870)
\end{array}\right)=\left(\begin{array}{cc}
\cos \theta_{P T} & \sin \theta_{P T} \\
-\sin \theta_{P T} & \cos \theta_{P T}
\end{array}\right)\left(\begin{array}{l}
\eta_{2, N} \\
\eta_{2, S}
\end{array}\right),
$$

with $\theta_{P T} \simeq-\frac{1}{2} \arctan \left[\frac{4 \sqrt{2} \alpha_{P T}}{2\left(m_{K_{2}(1770)}^{2}-m_{\pi_{2}(1660)}^{2}-\alpha_{P T}\right)}\right]$. According to the phenomenological study of Ref. [6], $\theta_{P T} \simeq-42^{\circ}$ : a surprisingly large and negative mixing (similar to the pseudoscalar sector) is realized, a fact that can be nicely explained by the axial anomaly being important in this (heterochiral) sector.

\section{Conclusions}

We have studied the role of the axial anomaly for light mesons. For the so-called "heterochiral" multiplets [4] (pseudoscalar and pseudotensor states), a large strange-nonstrange mixing is expected (a known fact for $\eta$ and $\eta^{\prime}$, some experimental evidence exists for pseudotensor mesons [6]). On the contrary, (axial-)vector and tensor mesons are "homochiral" and the anomaly does not affect the 
mixing: the isoscalar states are (almost) nonstrange and strange, respectively. Ongoing experimental activity at the JLab (e.g. Ref. [14]) can help to shed light on resonances between 1-2 GeV and hence on the role of the axial anomaly.

As recently shown, the axial anomaly can also be relevant in the baryonic sector. In particular, it can explain the large decay $N(1535) \rightarrow N \eta$ [15] and contribute to pion-nucleon scattering [16]. Moreover, the enigmatic pseudoscalar glueball [9] is also related to the axial anomaly and can be studied in the future.

Acknowledgments: the author thanks R. Pisarski and A. Koenigstein for cooperation. Financial support from the Polish National Science Centre NCN through the OPUS project no. $2015 / 17 / \mathrm{B} / \mathrm{ST} 2 / 01625$ is acknowledged.

\section{References}

[1] G. 't Hooft, Phys. Rept. 142, 357 (1986); D. J. Gross, S. B. Treiman and F. Wilczek, Phys. Rev. D 19, 2188 (1979); S. L. Adler, Phys. Rev. 177, 2426 (1969); J. S. Bell and R. Jackiw, Nuovo Cim. A 60, 47 (1969)

[2] J. Schechter, A. Subbaraman and H. Weigel, Phys. Rev. D 48, 339 (1993); T. Feldmann, P. Kroll and B. Stech, Phys. Rev. D 58, 114006 (1998); S. D. Bass and A. W. Thomas, Phys. Lett. B 634, 368 (2006); B. Borasoy and R. Nissler, Eur. Phys. J. A 26, 383 (2005)

[3] R. Escribano and J. M. Frere, JHEP 0506, (2005) 029; A. Bramon, R. Escribano and M. D. Scadron, Eur. Phys. J. C 7, 271 (1999); R. Escribano and J. Nadal, JHEP 0705, 006 (2007)

[4] F. Giacosa, A. Koenigstein and R. D. Pisarski, Phys. Rev. D 97, 091901 (2018)

[5] F. Giacosa, PoS Hadron 2017, 045 (2018)

[6] A. Koenigstein and F. Giacosa, Eur. Phys. J. A 52, 356 (2016)

[7] D. Parganlija, P. Kovacs, G. Wolf, F. Giacosa and D. H. Rischke, Phys. Rev. D 87, 014011 (2013); S. Janowski, F. Giacosa and D. H. Rischke, Phys. Rev. D 90, 114005 (2014)

[8] C. Rosenzweig, J. Schechter and C. G. Trahern, Phys. Rev. D 21, 3388 (1980); A. H. Fariborz, R. Jora and J. Schechter, Phys. Rev. D 72, 034001 (2005)

[9] W. I. Eshraim, S. Janowski, F. Giacosa and D. H. Rischke, Phys. Rev. D 87, 054036 (2013); W. I. Eshraim, S. Janowski, A. Peters, K. Neuschwander and F. Giacosa, Acta Phys. Polon. Supp. 5, 1101 (2012)

[10] G. Amelino-Camelia et al., Eur. Phys. J. C 68, 619 (2010)

[11] M. Tanabashi et al. (Particle Data Group), Phys. Rev. D 98, 030001 (2018)

[12] F. Divotgey, L. Olbrich and F. Giacosa, Eur. Phys. J. A 49, 135 (2013)

[13] F. Giacosa, T. Gutsche, V. E. Lyubovitskij and A. Faessler, Phys. Rev. D 72, 114021 (2005); L. Burakovsky and J. T. Goldman, Phys. Rev. D 57, 2879 (1998); V. Cirigliano, G. Ecker, H. Neufeld and A. Pich, JHEP 0306, 012 (2003)

[14] H. Al Ghoul et al. [GlueX Collaboration], AIP Conf. Proc. 1735, 020001 (2016); A. Rizzo [CLAS Collaboration], PoS CD 15, 060 (2016)

[15] L. Olbrich, M. Zétényi, F. Giacosa and D. H. Rischke, Phys. Rev. D 97, 014007 (2018)

[16] P. Lakaschus, J. L. P. Mauldin, F. Giacosa and D. H. Rischke, arXiv:1807.03735 [hep-ph] 